\title{
On the stability of the constant relative risk aversion (CRRA) utility under high degrees of uncertainty
}

\author{
Trino-Manuel Ñíguez ${ }^{\mathrm{a}, *}$, Ivan Paya ${ }^{\mathrm{b}}$, David Peel ${ }^{\mathrm{b}}$, Javier Perote ${ }^{\mathrm{c}}$ \\ a Department of Economics and Quantitative Methods, Westminster Business School, University of Westminster, London NW1 5LS, UK \\ ${ }^{\mathrm{b}}$ Department of Economics, Lancaster University Management School, Lancaster LA1 4YX, UK \\ ${ }^{\mathrm{c}}$ Department of Economics, University of Salamanca, 37007 Salamanca, Spain
}

\section{A R T I C L E I N F O}

\section{Article history:}

Received 13 January 2011

Received in revised form

16 November 2011

Accepted 13 December 2011

Available online 21 December 2011

\section{JEL classification:}

D80

\section{Keywords:}

Bayesian learning

Rational expectations

Semi-nonparametric distributions

\begin{abstract}
A B S T R A C T
Growth models under uncertainty and constant relative risk aversion (CRRA) utility are fragile in explaining consumers' choice, as equilibrium consumption is dependent on distributional assumptions. We show that, under semi-nonparametric distributions, general equilibrium models are stable, as the existence of expected utility is guaranteed.
\end{abstract}

(c) 2011 Elsevier B.V. All rights reserved.

\section{Introduction}

King et al. (1990) and Geweke (2001) highlight a number of difficulties in the application of rational expectations models to choice under uncertainty when the distribution of micro and macro aggregates is heavy tailed. They show that, when a Student's $t$-distribution rather than log-normality is assumed, the constant relative risk aversion (CRRA) expected utility ( $E U$ ) model does not have a solution leading to operational problems in the theory of choice. Further, Geweke notes that, even when lognormality is assumed, the $E U$ model does not have a solution regardless of the priors from which Bayesian learning begins. More generally, Geweke (2001) shows that general equilibrium models which embody rational expectations are quite fragile with respect to different distributional assumptions (which are often non-distinguishable on econometric grounds) and infinitesimal changes in over-time marginal rates of substitution which can lead to different equilibrium paths with quite different properties (see also Pesaran et al., 2007). Yoon (2004) also shows that similar fragility applies to a standard asset pricing model when

\footnotetext{
* Corresponding author. Tel.: +44 2079115000; fax: +44 2079115839.

E-mail addresses: t.m.niguez@wmin.ac.uk (T.-M. Ñíguez), i.paya@lancaster.ac.uk (I. Paya),d.peel@lancaster.ac.uk (D. Peel), perote@usal.es (J. Perote)
}

the endowment follows a stochastic unit root process. In this letter, we propose a solution to this problem. We assume that the logarithms of macroeconomic variables (e.g. log-consumption) follow a semi-nonparametric (SNP) density. The assumption of a SNP distribution for random log-consumption provides stability to general equilibrium models as the expected CRRA utility exists under Bayesian learning for any value of the over-time marginal rate of substitution. The rest of the letter is structured as follows. In Section 2, we describe the properties of the SNP probability distribution. In Section 3, we extend the results on $E U$ under CRRA assuming SNP densities, and also show that the $E U$ model is well defined under Bayesian updating for this density. The final section (Section 4) provides a brief conclusion. An empirical application and proofs of log-SNP properties are provided in Appendices A and $\mathrm{B}$, respectively.

\section{The SNP distributions}

This section describes properties of the SNP probability density function (pdf hereafter), which will be useful throughout the paper.

Proposition 1 (Cramér, 1925). Let $x$ be a continuous random variable distributed according to a certain pdf $f(x)$, which has a continuous derivative such that

$$
\int_{-\infty}^{\infty}\left(\frac{d f(x)}{d x}\right)^{2} e^{\frac{1}{2} x^{2}} d x<\infty \text { and } f(x) \underset{|x| \rightarrow \infty}{\rightarrow} 0 .
$$


Then, $f(x)$ can be expanded formally on a (infinite) series of derivatives of the standard normal density, denoted as $\phi(x)$, as follows:

$f(x)=\sum_{s=0}^{\infty} \kappa_{s} H_{s}(x) \phi(x)$,

where $\kappa_{s}=\frac{1}{s !} \int_{-\infty}^{\infty} H_{s}(x) \phi(x) d x$, and $H_{s}(x)$ is the sth-order Chebyshev-Hermite polynomial, which can be defined by the identity in Eq. (3), ${ }^{1}$

$\frac{d^{s} \phi(x)}{d x^{s}}=(-1)^{s} H_{s}(x) \phi(x), \quad \forall s \geq 1$.

Proposition 1 allows one to define a general family of SNP distributions, $g(x ; \delta), \delta \in \mathbb{R}^{n}, \delta=\left(\delta_{1}, \ldots, \delta_{n}\right)$, which can approximate any pdf to any degree of accuracy depending on the truncation order $n .^{2}$

$g(x ; \delta)=\left[1+\sum_{s=1}^{n} \delta_{s} H_{s}(x)\right] \phi(x) \simeq f(x)$.

The rationale behind this density expansion lies in the properties of the Chebyshev-Hermite polynomials, which form an orthonormal basis with respect to the weight function $\phi(x)$ (see Abramowitz and Stegun, 1972, or Kendall and Stuart, 1977, for further details).

$$
\begin{aligned}
& \int_{-\infty}^{\infty} H_{s}(x) H_{r}(x) \phi(x) d x= \begin{cases}0, & s \neq r \\
s !, & s=r .\end{cases} \\
& \frac{d H_{s}(x)}{d x}=s H_{s-1}(x), \quad \forall s \geq 1 .
\end{aligned}
$$

Based on these properties, other characterizations of the SNP distributions can be obtained straightforwardly; for example, Eq. (7) gives the cumulative distribution function (cdf hereafter), and Eq. (8) is the moment-generating function (mgf hereafter) (see Proofs 1 and 2 in Appendix B):

$$
\begin{aligned}
G_{x}(a) & =\int_{-\infty}^{a} g(x ; \delta) d x \\
& =\int_{-\infty}^{a} \phi(x) d x-\phi(a) \sum_{s=1}^{n} \delta_{s} H_{s-1}(a), \\
M_{x}(t) & =E\left[e^{t x}\right] \\
& =\int_{-\infty}^{\infty} e^{t x} g(x ; \delta) d x=e^{t^{2} / 2}\left[1+\sum_{s=1}^{n} \delta_{s} t^{s}\right] .
\end{aligned}
$$

In addition, all order SNP distribution moments exist and are functions of the density parameters. For example, the first four central moments of the SNP pdf are $E[x]=\delta_{1}, E\left[x^{2}\right]=1+$ $2 \delta_{2}, E\left[x^{3}\right]=3 \delta_{1}+6 \delta_{3}$, and $E\left[x^{4}\right]=3+12 \delta_{2}+24 \delta_{4}$, and the distribution has zero mean and unit variance if $\delta_{1}=\delta_{2}=0$, with $\delta_{3}$ and $\delta_{4}$ capturing the skewness and excess kurtosis of the distribution, respectively (see Proof 3 in Appendix B).

\section{The CRRA utility under SNP distributions}

This section extends the $E U$ results under CRRA by assuming SNP-distributed microeconomic and macroeconomic variables.

\footnotetext{
1 This is the so-called Gram-Charlier series of Type A. By convention, it is usually assumed that $H_{0}(x)=1$.

2 The truncation of the expansion involves positivity issues, which can be addressed through either pdf reformulations (Gallant and Nychka, 1987) or parametric constraints (Jondeau and Rockinger, 2001). In most cases, the use of maximum likelihood estimation techniques for empirical purposes does not require any restriction to obtain well-defined densities at the optimal $\delta_{s}$ parameters Appendix A provides details of log-SNP density estimation for consumption growth data. On the other hand, note that without loss of generality we consider $\delta_{0}=1$.
}

We show that, in contrast to the Student's $t$-distribution, a logSNP pdf is valid for a wider range of possibilities, thus providing consistency to rational expectations models in regards to heavytailed distributional assumptions.

Definition 1. Let $x=\log (z)$ be a random variable with pdf $f(x)$, and $\alpha$ a strictly positive parameter. We define the $E U$ of $z>0$ under CRRA as

$E U(z)= \begin{cases}\log (z) & \text { if } \alpha=1 \\ (1-\alpha)^{-1} E\left[z^{1-\alpha}\right] & \text { if } \alpha \neq 1 .\end{cases}$

The $E U$ function defined above is valid for any strictly nonnegative random variables, e.g. consumption, provided that the following hold.

1. The function $f(x)$ is known.

2. The $\operatorname{mgf}, M_{x}(t)=E\left[e^{t x}\right]$, exists for all $t=1-\alpha$ and $\alpha \neq 1$.

Then, under assumptions 1 and 2, it is straightforward to show that $E U(z)$ exists for all $\alpha \neq 1$, and it is given by

$E U(z)=(1-\alpha)^{-1} E\left[e^{(1-\alpha) x}\right]=(1-\alpha)^{-1} M_{x}(1-\alpha)$.

The most common distributional assumption in the literature on choice theory for the pdf of $z$ is the log-normal one, i.e. $x \sim$ $N\left(\mu, \sigma^{2}\right)$, as it delivers estimates with almost surely known properties. From a pragmatic viewpoint, improvements in model reliability and theory predictions can be achieved assuming alternative densities to the log-normal which may better capture features of the data such as heavy tails. A straightforward way of assuming a heavy-tailed distribution for $x$ is by means of the Student's $t$-distribution with $v$ degrees of freedom, i.e. $x \sim$ $t\left(\mu, \sigma^{2} ; v\right)$. But under this assumption the CRRA utility is shown not to be well defined, since the mgf of the Student's $t$-distribution fails to exist for any $t \neq 0$. This case is examined in Geweke (2001), which points out the limitations of the CRRA utility when the log-normal assumption is relaxed to incorporate a more realistic heavy-tailed pdf (see also Kendall and Stuart, 1977, p. 60). If the period utility function is bounded and the $E U$ is guaranteed to exist, Geweke's result would not apply. This is the case for some classes of hyperbolic absolute risk aversion (HARA) utility functions (e.g. Cogley, 2009). It is also possible to obtain the existence of $E U$ even though period utility is unbounded, for instance, in the case of a 'finite-state' economy (see Cogley and Sargent, 2008).

Definition 2. We say that variable $z>0$ is log-SNP distributed if the pdf of the variable $x=\log (z)$ is that in Eq. (4) above, $x \sim \operatorname{SNP}(\boldsymbol{\delta})$. For that variable, and provided that $\alpha>0$, the $E U$ consistent with the CRRA hypothesis is defined as

$E U(z)$

$$
= \begin{cases}\log (z) & \text { if } \alpha=1 \\ (1-\alpha)^{-1} e^{(1-\alpha)^{2} / 2}\left[1+\sum_{s=1}^{n} \delta_{s}(1-\alpha)^{s}\right] & \text { if } \alpha \neq 1\end{cases}
$$

The $E U$ in Eq. (11) is well defined as all order moments of the SNP distribution and the mgf (Eq. (8)) exist. Consequently, the logSNP model appears useful as a method of generating solutions in $E U$ models when the distributions are assumed to exhibit heavy tails.

We also note that assumption 1 does not seem to be consistent under CRRA if the $E U$ is accomplished by Bayesian updating. This fact makes rational expectations models dependent not only on the distributional assumptions but also on the subjective distribution of the priors. In this case, Geweke (2001) argues that the $E U$ fails to 
exist even in the log-normal case and regardless of the priors from which Bayesian learning begins. ${ }^{3}$ Nevertheless, conditions can be found for which the $E U$ is well defined under Bayesian updating. In particular, Definition 3 describes the $E U$ for log-SNP nonnegative random variables in a Bayesian framework.

Definition 3. Let $x=\log (z), z>0$, be a random variable with pdf $g(x ; \delta)$ (Eq. (4)), where the parameter vector $\delta$ is unknown and has subjective pdf $\varphi(\boldsymbol{\delta})$ with support $\mathbb{R}^{n}$. If $E(x)=\int \cdots \int_{\boldsymbol{\Delta}}$ $E[x \mid \delta] \varphi(\boldsymbol{\delta}) d \boldsymbol{\delta}_{1} \cdots d \boldsymbol{\delta}_{n}$ exists, then

$E U(z)= \begin{cases}E(x) & \text { if } \alpha=1, \\ (1-\alpha)^{-1} E_{\delta}\left[M_{x}(1-\alpha ; \delta)\right] & \text { if } \alpha \neq 1,\end{cases}$

since $M_{x}(1-\alpha ; \delta)$ exists for all $\delta \in \mathbb{R}^{n}$ and is finitely integrable with respect to $\delta$.

For example, if $x \mid(\boldsymbol{\delta}) \sim \operatorname{SNP}(\boldsymbol{\delta})$ and $d_{i} \sim N\left(\overline{\boldsymbol{\delta}_{i}}, \bar{q}\right) \forall i=1$, $\ldots, n,{ }^{4}$ then $x \sim \operatorname{SNP}(\bar{\delta})$. In this case, it follows that

$E U(z)$

$$
= \begin{cases}\overline{\delta_{1}}, & \text { if } \alpha=1, \\ (1-\alpha)^{-1} e^{(1-\alpha)^{2} / 2}\left[1+\sum_{s=1}^{n} \overline{\delta_{s}}(1-\alpha)^{s},\right] & \text { if } \alpha \neq 1 .\end{cases}
$$

The example above illustrates the fact that the log-SNP pdf may be used to overcome the fragility of rational expectations models under CRRA utility and reasonable assumptions about the subjective distribution of SNP pdf parameters.

\section{Conclusion}

Recent evidence in the literature (see the seminal paper of Geweke, 2001) shows that traditional equilibrium models of growth under the common assumptions of CRRA utility and rational expectations may not be well defined when macroeconomic variables exhibit heavy tails and/or learning is accomplished by Bayesian updating. In these cases, the existence of expected utility becomes very fragile with respect to the distributional assumptions leading to a non-operational theory of choice, particularly when the model tries to embody high levels of economic uncertainty through heavy-tailed distributions.

In this paper, we follow an alternative approach to that of Geweke (2001) to recover an operational theory of choice. We propose the replacement of the traditional assumption of log-normality by (possibly heavy-tailed and skewed) logsemiparametrically distributed random macroeconomic variables (e.g. log-consumption). The advantages of this approach are twofold. (a) As in Geweke (2001), it provides stability to the existence of the agents' expected utility under CRRA and Bayesian learning, and (b) it provides the agents with the possibility to better identify the probabilities of extreme events which may occur under economic situations of high uncertainty. Thus, while Geweke (2001) provides a solution to the stability of expected utility

\footnotetext{
3 See Examples 4 and 5 in Geweke (2001). It is worth mentioning that recent contributions in the area of asset pricing have circumvented the problems with Bayesian learning highlighted by Geweke in different ways. Bidarkota et al. (2009) use a subfamily of $\alpha$-stable distributions (the ones with maximal negative skewness of -1 ) to provide an operational theory under uncertainty for that particular case. Bakshi and Skoulakis (2010) develop the model of Weitzman (2007) further, and obtain a model that (with subjective expectations) yields a well-defined expected utility and a finite moment-generating function of the predictive distribution of consumption growth.

4 For example, given a prior $d_{i} \sim N\left(d_{i}, q\right)$ and $T$ i.i.d. observations $x_{1}, \ldots, x_{T}$, $\bar{q}^{-1}=\underline{q}^{-1}+T$ and $\overline{d_{i}}=\bar{q}\left(\underline{q}^{-1} \underline{d_{i}}+T \bar{x}\right)$. See Example 3 in Geweke (2001).
}

through modifications of the agents' Bayesian learning process still under log-normality, we focus on the whole distributional assumption for log-consumption. We argue that our approach allows us to recover an even more operational theory of choice with CRRA utility, Bayesian learning and, in particular, heavy-tailed distributed random consumption.

\section{Acknowledgments}

Javier Perote acknowledges financial support from the Spanish Ministry of Education and Castilla and León Government under grants SEJ2007-66592-C03-03 and SA218A11-1, respectively. Ivan Paya acknowledges financial support from the Spanish Ministry of Education and Science research project ECO2008-05721/ECON.

\section{Appendix A}

This appendix presents an empirical application of the logSNP density. It provides details of the estimation of the density parameters and assesses its goodness of fit in relation to the lognormal and Student's $t$ densities used as benchmarks. The data used are (monthly) percentage gross consumption growth $\left(c_{t}\right)$ computed from an observed sample of (monthly) US personal consumption expenditure, $c_{t}=1+\log \left(C_{t} / C_{t-1}\right),, t=1, \ldots, T$, over the period November 1961 to October 2011 for a total of $T=598$ observations. $c_{t}$ presents sample values of mean and skewness equal to 1.0175 and 0.2304 , respectively, and sample standard deviation of 0.0256 and, high kurtosis of 12.10 biased upwards by the presence of three very extreme positive and negative observations. The Jarque-Bera test rejects the null of normality at least at the $0.001 \%$ confidence level.

The log-SNP density is estimated sequentially up to a truncation order of $n=4$. Thus, the log-likelihood function for one observation corresponding to $c_{t}$ distributed as a $\log \operatorname{SNP}\left(\mu, \sigma^{2}, \boldsymbol{\delta}\right)$ truncated at the fourth moment is given by $l_{t}=-\frac{1}{2} \ln \left(2 \pi \sigma^{2} c_{t}^{2}\right)-$ $\frac{1}{2}\left(\frac{\ln \left(c_{t}\right)-\mu}{\sigma}\right)^{2}+\ln \left[1+\sum_{s=1}^{4} \delta_{s} H_{s}\left(\frac{\ln \left(c_{t}\right)-\mu}{\sigma}\right)\right]$. Sequential estimation starts with the simplest nested density, i.e. the log-normal, and recursively adds $\delta_{i}$ parameters whose starting values are selected consistently with the sample moments. Maximum likelihood (ML) estimation of the log-SNP density is obtained smoothly following this method. ${ }^{5}$ Table A. 1 reports the ML estimates of the aforementioned densities for $c_{t}$. The estimation results show that all models capture very well the mean and standard deviation of the series, underestimate the kurtosis, and mixed results are found for the skewness. Overall, the $\log$-SNP $(n=4)$ density provides more accurate estimates of all moments than the other considered densities. On the other hand, the best goodness of fit according to the AIC is provided by the $\log -\mathrm{SNP}^{(\mathrm{c})}(n=4)$ (restricted to $\delta_{1}=0$ ) density. Note that the Student's $t$-distribution AIC is not comparable with those of log-SNP and log-normal. The Student's $t$ estimated kurtosis, $\widehat{k u}=3(\widehat{v}-2) /(\widehat{v}-4)$, is not displayed, as the optimal value of the degrees of freedom parameter, $v$, converges to the limit of 4 , which is the lower bound needed for the existence of the first four moments of the density. This Student's $t$ estimation case usually occurs when the target empirical distribution is leptokurtic with a pronounced peak in the center. This is a documented result in the economic literature, which has given place to the search for alternative heavy-tailed distribution functions such as, for example, SNP densities. ${ }^{6}$

\footnotetext{
5 See León et al. (2005) and Ñíguez and Perote (in press) for further details on optimal truncation and alternative estimation methods for SNP densities.

6 See Mauleón and Perote (2000).
} 
Table A.1

Estimation results.

\begin{tabular}{|c|c|c|c|c|c|}
\hline & $\log -\operatorname{SNP}(n=4)$ & $\log -\mathrm{SNP}^{(\mathrm{c})}(n=4)$ & $\log -\operatorname{SNP}(n=2)$ & log-normal & Student's $t$ \\
\hline$\widehat{\mu}$ & $0.0124(8.40)$ & $0.0174(17.7)$ & $-0.0082(-6.02)$ & $0.0171(16.6)$ & $1.0152(128.4)$ \\
\hline$\widehat{\sigma}$ & $0.0250(33.3)$ & $0.0255(28.6)$ & $0.0260(29.3)$ & $0.0251(35.1)$ & $0.0230(25.4)$ \\
\hline$\widehat{\delta}_{1}$ & $0.1867(4.26)$ & $0.0000(\cdot)$ & $0.9745(48.9)$ & & \\
\hline$\widehat{\delta}_{2}$ & $0.0208(0.53)$ & $-0.0473(-1.49)$ & $0.4423(9.04)$ & & \\
\hline$\widehat{\delta}_{3}$ & $-0.0049(-0.15)$ & $0.0661(2.59)$ & & & \\
\hline$\widehat{\delta}_{4}$ & $0.1191(9.48)$ & $0.1026(8.46)$ & & & \\
\hline$\widehat{v}$ & & & & & $4.0000(\cdot)$ \\
\hline$\widehat{m}$ & 1.0175 & 1.0179 & 1.0175 & 1.0175 & 1.0152 \\
\hline$\widehat{s . d .}$ & 0.0256 & 0.0249 & 0.0253 & 0.0256 & 0.0230 \\
\hline$\widehat{s k}$ & 0.1412 & 0.6333 & -0.6657 & 0.0755 & 0.0000 \\
\hline$\widehat{k u}$ & 5.7550 & 6.1115 & 5.2522 & 3.0101 & $+\mathrm{INF}$ \\
\hline $\log L$ & 1428.7 & 1418.5 & 1395.5 & 1343.2 & 1433.0 \\
\hline AIC & -4.4758 & -4.7275 & -4.6541 & -4.4857 & -4.7828 \\
\hline
\end{tabular}

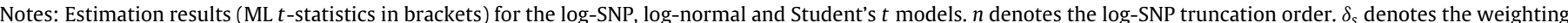

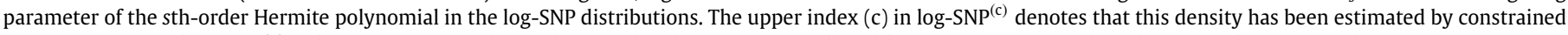

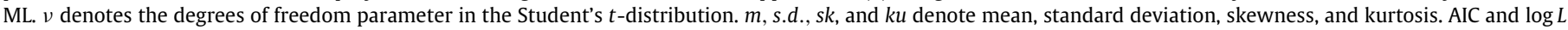
denote Akaike Information Criterion and log-likelihood, respectively.

\section{Appendix B}

Proof 1. The cdf of the SNP distribution is given by

$$
\begin{aligned}
G_{x}(a) & =\int_{-\infty}^{a} g(x ; \delta) d x \\
& =\int_{-\infty}^{a} \phi(x) d x+\sum_{s=1}^{n} \delta_{s} \int_{-\infty}^{a} H_{s}(x) \phi(x) d x \\
& =\int_{-\infty}^{a} \phi(x) d x-\left.\sum_{s=1}^{n} \delta_{s} H_{s-1}(x) \phi(x)\right|_{-\infty} ^{a} \\
& =\int_{-\infty}^{a} \phi(x) d x-\phi(a) \sum_{s=1}^{n} \delta_{s} H_{s-1}(a) .
\end{aligned}
$$

Since $\lim _{x \rightarrow \pm \infty} H_{s}(x) \phi(x)=0 \forall s \geq 1$, we obtain

$$
\begin{aligned}
\int H_{s}(x) \phi(x) d x & =\int(-1)^{s} \frac{d^{s} \phi(x)}{d x^{s}} d x_{t} \\
& =(-1)^{s} \frac{d^{s-1} \phi(x)}{d x^{s-1}}=(-1)^{s}(-1)^{s-1} H_{s-1}(x) \phi(x) \\
& =-H_{s-1}(x) \phi(x) .
\end{aligned}
$$

Proof 2. The mgf of the SNP distribution is given by

$$
\begin{aligned}
M_{x}(t)= & \int_{-\infty}^{\infty} e^{t x} g(x ; \delta) d x=\int_{-\infty}^{\infty} e^{t x} \phi(x) d x \\
& +\sum_{s=1}^{n} \delta_{s} \int_{-\infty}^{\infty} e^{t x} H_{s}(x) \phi(x) d x \\
= & e^{t^{2} / 2}+\sum_{s=1}^{n} \delta_{s}\left[-\left.e^{t x} H_{s-1}\left(x_{t}\right) \phi\left(x_{t}\right)\right|_{-\infty} ^{\infty}\right. \\
& \left.+\int_{-\infty}^{\infty} t e^{t x} H_{s-1}\left(x_{t}\right) \phi\left(x_{t}\right) d x\right] \\
= & e^{t^{2} / 2}+\sum_{s=1}^{n} \delta_{s} \int_{-\infty}^{\infty} t^{s} e^{t x} \phi(x) d x \\
= & e^{t^{2} / 2}\left[1+\sum_{s=1}^{n} \delta_{s} t^{s}\right] .
\end{aligned}
$$

Integrating by parts and taking into account the property in (6) and $e^{t x} H_{s}(x) \phi(x) \underset{x \rightarrow \pm \infty}{\rightarrow} 0 \forall s \geq 1$, we have

$u=e^{t x} \Longrightarrow d u=t e^{t x} d x$

$d v=H_{s}(x) \phi(x) d x \Longrightarrow v=-H_{s-1}(x) \phi(x)$.

Proof 3. The first four central moments of the SNP distributions are

$$
\begin{aligned}
& \left.\frac{d M_{x}(t)}{d t}\right|_{t=0}=\left[e^{t^{2} / 2}\left\{t+\sum_{s=1}^{n} \delta_{s}\left(t^{s+1}+s t^{s-1}\right)\right\}\right]_{t=0}=\delta_{1} . \\
& \left.\frac{d^{2} M_{x}(t)}{d t^{2}}\right|_{t=0}=\left[e ^ { t ^ { 2 } / 2 } \left\{1+t^{2}+\sum_{s=1}^{n} \delta_{s}\left(t^{s+2}+(2 s+1) t^{s}\right.\right.\right. \\
& \left.\left.\left.+s(s-1) t^{s-2}\right)\right\}\right]_{t=0}=1+2 \delta_{2} . \\
& \left.\frac{d^{3} M_{x}(t)}{d t^{3}}\right|_{t=0} \\
& =\left[e ^ { t ^ { 2 } / 2 } \left\{3 t+t^{3}+\sum_{s=1}^{n} \delta_{s}\left(t^{s+3}+3(s+1) t^{s+1}\right.\right.\right. \\
& \left.\left.\left.+3 s^{2} t^{s-1}+s(s-1)(s-2) t^{s-3}\right)\right\}\right]_{t=0} \\
& =3 \delta_{1}+6 \delta_{3} . \\
& \left.\frac{d^{4} M_{x}(t)}{d t^{4}}\right|_{t=0} \\
& =\left[e ^ { t ^ { 2 } / 2 } \left\{3+6 t^{2}+t^{4}+\sum_{s=1}^{n} \delta_{s}\left[t^{s+4}+3(s+1) t^{s+2}\right.\right.\right. \\
& +\left(6 s^{2}+6 s+3\right) t^{s}+2 s(s-1)(2 s-1) t^{s-2} \\
& \left.\left.\left.+s(s-1)(s-2)(s-3) t^{s-4}\right]+\right\}\right]_{t=0} \\
& =3+12 \delta_{2}+24 \delta_{4} \text {. }
\end{aligned}
$$

\section{References}

Abramowitz, M., Stegun, I.A., 1972. Orthogonal polynomials. In: Abramowitz, M., Stegun, I.A. (Eds.), Handbook of Mathematical Functions. Dover, New York. 
Bakshi, G., Skoulakis, G., 2010. Do subjective expectations explain asset pricing puzzles. Journal of Financial Economics 98, 462-477.

Bidarkota, P.V., Dupoyet, B.V., McCulloch, J.H., 2009. Asset pricing with incomplete information and fat tails. Journal of Economic Dynamics and Control 33, $1314-1331$.

Cogley, T., 2009. Is the market price of risk infinite? Economics Letters 102, 13-16.

Cogley, T., Sargent, T.J., 2008. The market price of risk and the equity premium: a legacy of the great depression? Journal of Monetary Economics 55, 454-476.

Cramér, H., 1925. On some classes of series used in mathematical statistics. In: Proceedings of the Sixth Scandinavian Congress of Mathematicians, pp. 399-425.

Gallant, A.R., Nychka, D.W., 1987. Seminonparametric maximum likelihood estimation. Econometrica 55, 363-390.

Geweke, J., 2001. A note on some limitations of CRRA utility. Economics Letters 71, 341-345.

Jondeau, E., Rockinger, M., 2001. Gram-Charlier densities. Journal of Economic Dynamics and Control 25, 1457-1483.
Kendall, M., Stuart, A., 1977. The Advanced Theory of Statistics, vol. 1. Charles Griffing, London.

King, R., Plosser, C.I., Rebelo, S.T., 1990. Production, growth and business cycles: technical appendix. University of Rochester Mimeo.

León, A., Rubio, G., Serna, G., 2005. Autoregressive conditional volatility, skewness and kurtosis. The Quarterly Review of Economics and Finance 45, 599-618.

Mauleón, I., Perote, J., 2000. Testing densities with financial data: an empirical comparison of the Edgeworth-Sargan density to the Student's $t$. The European Journal of Finance 6, 225-239.

Ñíguez, T.M., Perote, J., 2012. Forecasting heavy-tailed densities with positive Edgeworth and Gram-Charlier expansions. Oxford Bulletin of Economics and Statistics, in press (doi:10.1111/j.1468-0084.2011.00663.x).

Pesaran, H., Pettenuzzo, D., Timmermann, A., 2007. Learning, structural instability and present value calculations. Econometric Reviews 26, 253-288.

Weitzman, M., 2007. Subjective expectations and asset-return puzzles. American Economic Review 97, 1102-1130.

Yoon, G., 2004. On the existence of expected utility with CRRA under STUR. Economics Letters $83,219-224$. 